\title{
Zur Neuausgabe von Dietrich Bonhoeffers Werken
}

Zur Zeit findet wohl kein deutschsprachiger Theologe so viel Beachtung und Resonanz in der Weltchristenheit wie Dietrich Bonhoeffer. Die Verbreitung seiner Bücher macht das sichtbar. Deren Gesamtauflage hat die Zahl einer halben Million längst überstiegen. „Nachfolge“ und "Widerstand und Ergebung“ wurden in mehr als 16 Sprachen übersetzt, „Gemeinsames Leben" liegt in der 20. Auflage vor. Die konfessionellen Grenzen sind überschritten, unter Katholiken ist Bonhoeffer ebenso bekannt wie unter Protestanten; die verschiedensten Frömmigkeitsrichtungen und kirchenpolitischen Gruppierungen finden Zugang zu ihm. Viele achten ihn als Blutzeugen des christlichen Glaubens.

In weiten Teilen der Ökumene steht Bonhoeffers Name für die Gruppen und Personen, die den Kirchenkampf unter der nationalsozialistischen Gewaltherrschaft entschieden und mit höchstem Einsatz geführt haben. Der Weg vom klaren Widerspruch aus theologischen Gründen in die umfassende Opposition, in den Ungehorsam gegen Unrechtsmaßnahmen der Partei und des Staates, in den Kampf für die bekennende Kirche und für die gejagten Juden und schließlich in die Konspiration mit dem Ziel, Recht und Freiheit in Deutschland und in Europa wiederherzustellen, dieser Weg ist von Bonhoeffer unter manchen schweren Belastungen, aber schließlich unbeirrt im Glaubensgehorsam gegangen worden. Mit kirchlichen Widerstandsbewegungen in den von Deutschland besetzten Ländern, besonders in Skandinavien, hielt er Kontakt. $\mathrm{Zu}$ verschiedenen Zeitpunkten, an denen der deutsche Widerstand Friedensbedingungen mit England nach einem Sturz Hitlers auszuhandeln versuchte, hat er als ein wichtiger Vermittler gedient. Er gehörte zu denen, die die Absetzung beziehungsweise die Beseitigung Hitlers als unabdingbare Voraussetzung für die Beendigung des Zweiten Weltkrieges erkannten und vorbereiteten. Männern des Widerstandes wurde er zum Gewissensberater. 
Ihre Probleme, ihre Konflikte hat er ethisch durchdacht und theologisch verarbeitet. Dies tat er nicht aus sicherer Distanz, sondern unter Übernahme des vollen Risikos für sein Leben, für seine Familie und seine Freunde. Jeder seiner Texte zeugt dafür, daß Bonhoeffer - in aller Anfechtung - beharrlich den Weg der Nachfolge suchte; jeder seiner Texte zeigt aber auch den nüchternen Sinn für die Wirklichkeit, mit der er zu tun hatte. Gerade diese enge Verbindung, die ,Einheit von Biographie und Theologie' (Eberhard Bethge, Christian Gremmels), macht die Begegnung mit Bonhoeffer lohnend - besonders für die, die es schwer haben, als Christen unter einem Unrechtsregime den Weg zu finden, den ihnen der Glaube gebietet.

Aber die nahezu singuläre Verbindung von Theologie und Widerstand war nach seiner Hinrichtung am Galgen in Flossenbürg am 9. April 1945 auch vielen ein Hindernis, die Bedeutung seines Werkes zu erkennen. Insbesondere im deutschen evangelischen Kirchentum ist - anders als in vielen Teilen der Ökumene - das Verhältnis von Glaube und Politik so umstritten und ungeklärt, daß schon Bonhoeffers Beteiligung am Widerstand ihn für manche verdächtig und zum kirchlichen Außenseiter machte. Nach 1945 hat zunächst keine kirchliche, keine akademische oder staatliche Institution die Verbreitung seines Werkes gefördert. Ohne den Freund Eberhard Bethge wären weite Teile von Bonhoeffers Werk in Vergessenheit geraten. Die zahlreichen hinterlassenen Manuskripte, Briefe und Aufzeichnungen wären wohl kaum veröffentlicht worden. Der Chr. Kaiser Verlag wagte es, 1949 die von Bethge redigierten unvollendeten Manuskripte zu Bonhoeffers „Ethik“ herauszubringen. 1951 folgte „Widerstand und Ergebung“. Mit diesem Buch war der Durchbruch in die Öffentlichkeit erzielt. Wegen der unerwartet starken und vielfältigen Resonanz, wegen der zahlreichen Rückfragen nach Bonhoeffers Leben und Werk konnte Bethge jetzt darangehen, die vielen verstreuten Briefe, Aufsätze, Dokumente, Predigten und anderen Hinterlassenschaften in Jahrzehnten zu sammeln und von 1958 an in den „Gesammelten Schriften“ beim Chr. Kaiser Verlag herauszubringen. Der erste Band präsentierte Bonhoeffers Wirken in der Ökumene und brachte damit zum Ausdruck, wie sehr es Bonhoeffer am Herzen gelegen hat, daß die Christenheit aus ihrer 
Zersplitterung zur Einheit der Kirche Jesu Christi finde. Drei weitere nach thematischen und biographischen Gesichtspunkten zusammengestellte Bände und schließlich zwei Ergänzungsbände folgten. Natürlich konnte die Anordnung der sechs Bände nur provisorisch sein; sie konnte eine strenge Chronologie schon deshalb nicht einhalten, weil ständig neue Materialien aufgetaucht waren. Jørgen Glenthøj gab ergänzend den Band „Dokumente zur Bonhoeffer-Forschung 1928-1945“ bei Chr. Kaiser 1969 heraus. Aber auch das bedeutete keinen Abschluß. Noch 1985 wurden unter anderem in der Schroederbank in London Bonhoeffer-Briefe gefunden. Der Nachlaß von Julius Rieger, dem Pfarrerkollegen Bonhoeffers in London, wird erst jetzt zugänglich. Aus Ungarn sind kürzlich Nachschriften aus Bonhoeffers Kollegs in den Jahren 1932/33, darunter die einzige aus Bonhoeffers Hegel-Seminar, zur Verfügung gestellt worden. Die Briefe Bonhoeffers an seine Braut Maria von Wedemeyer sind freilich einstweilen der Forschung und der Öffentlichkeit noch nicht zugänglich. Alles in allem zeigt sich, daß eine Gesamtausgabe der Werke Dietrich Bonhoeffers im strengen Sinne auch jetzt noch nicht möglich ist.

Dennoch erschien es 1981 dem Internationalen Bonhoeffer Komitee Sektion Bundesrepublik Deutschland in Absprache mit dem Bonhoeffer Komitee beim Bund der Evangelischen Kirchen in der Deutschen Demokratischen Republik geboten, eine wissenschaftliche Neuausgabe $\mathrm{zu}$ erarbeiten. Wichtiges bisher unveröffentlichtes Material geht in sie ein. Aber es wird darauf verzichtet, alle von Bonhoeffer erhaltenen Aufzeichnungen wiederzugeben. Da der Gesamtnachlaß im Bundesarchiv in Koblenz auf Mikro-Fiches archiviert und ein Nachlaßverzeichnis hergestellt wird, ist künftig das ganze Material, mit Ausnahme der Briefe an Bonhoeffers Braut, für die Forschung unter Archivbedingungen zugänglich. Die Werkausgabe braucht daher Texte, die für nur wenige Leser von Interesse sein werden, nicht abzudrucken, wenn auch auf sie an ihrem chronologischen und sachlichen Ort hingewiesen wird. Das gilt zum Beispiel für bloße Stoffsammlungen, die sich der Student Bonhoeffer aus Kompendien zusammengestellt hat.

Die Ausgabe der Werke jetzt in Angriff zu nehmen, ist dringlich, weil der Kreis der lebenden Zeitgenossen, Bekann- 
ten, Verwandten und Freunde Dietrich Bonhoeffers von Jahr zu Jahr kleiner wird. Möglichst viele Auskünfte aus diesem Kreis sollen in der kommentierten Werkausgabe verarbeitet werden. Diese Ausgabe soll Bonhoeffers Werke in textkritisch überprüfter Form mit knappen Sachkommentierungen nach heutigem Kenntnisstand darbieten. Eine möglichst weitgehende formale Übereinstimmung in der Bearbeitung aller Bände wird angestrebt.

Es sind insgesamt 16 Bände mit rund 8000 Seiten vorgesehen. Als Band 1 bis 8 werden die von Bonhoeffer selbst publizierten und die drei von Eberhard Bethge posthum zusammengestellten Bücher vorgelegt. Da zwei der letzteren eine bedeutende eigene Wirkungsgeschichte haben, wäre es ungut gewesen, in ihnen enthaltene Texte in die, biographischen' Bände 9 bis 16 einzuordnen, was unter formalen Gesichtspunkten durchaus denkbar gewesen wäre, so zum Beispiel den Inhalt von „Widerstand und Ergebung “ in den Band 16 „Konspiration und Haft 1939-1945“.

Die Bände 9 bis 16, die chronologisch acht Zeitabschnitte berücksichtigen, werden in sich drei im ganzen übereinstimmende, im einzelnen aber leicht variierende Teile enthalten: einen ersten mit Briefen, Tagebüchern, Dokumenten, einen zweiten mit Ausarbeitungen, Aufsätzen, Gutachten, einen dritten mit Predigten, Meditationen, geistlichen Texten. Innerhalb dieser drei Sachgruppen wird streng chronologisch verfahren. Bei einer solchen Gliederung, die literarische Genera und chronologisches Schema kombiniert, wird für den Leser wie für den Forscher die größtmögliche Übersichtlichkeit erreicht. Einerseits bleibt zum Beispiel die Beziehung zwischen den einzelnen Briefen erhalten, andererseits kann der Leser schnell erkennen, was Bonhoeffer zur gleichen Zeit in Tagebuchnotizen, Konferenzvorbereitungen und Predigten beschäftigt hat.

Die textkritischen Probleme sind für die einzelnen Bände höchst unterschiedlicher Natur. Darüber geben die Vorworte der Einzelbände Auskunft. Bei der Kommentierung streben die Bandherausgeber möglichst weitgehende Zurückhaltung in der theologischen und historischen Interpretation an. Dem Leser soll erspart werden, sich gegen eine bevormundende Auslegung im Sinne theologischer oder kirchlicher Richtungen oder sub- 
jektiver Herausgeberurteile wehren zu müssen. Doch neben den unentbehrlichen Sachinformationen werden Verweise auf vermutlich zugrundeliegende theologische, philosophische oder sonstige Texte und auch auf vergleichbare Äußerungen Bonhoeffers an anderer Stelle aufgenommen. Sie sollten nicht aus puristischem Objektivismus dem Leser vorenthalten werden, zumal sie jederzeit überprüfbar sind. Dabei ist nicht vergessen worden, daß schon die Auswahl dessen, was man mit einer Anmerkung versieht, was man also für erklärungs- oder verweisbedürftig hält, vom persönlichen Urteil des Herausgebers - und derer, mit denen er sich berät - abhängt. Allzugroße Zurückhaltung macht freilich die Anmerkungen langweilig, so daß jeder Herausgeber den rechten Ausgleich zwischen Objektivität und Deutlichkeit seiner Kommentierung finden muß.

Um dem Leser einen zuverlässigen Einblick in die Verfahrensweise des Bandherausgebers zu geben, legt dieser im Nachwort Überlegungen zu seiner Bearbeitung und Interpretation des Textes vor. Hier kann er einen größeren Spielraum für persönliche Auffassungen beanspruchen. Das editorische Vorwort hingegen zielt auf möglichst straffe Information über die Entstehungsgeschichte des Textes und die Regeln für die Erarbeitung der vorgelegten Textgestalt.

Die Herausgeber sahen sich verpflichtet, nicht nur an den Leser zu denken, der mit deutschem Kirchentum, deutscher Theologie und Geschichte vertraut ist. Macht man mit der Erkenntnis Ernst, daß Bonhoeffer heute der Weltchristenheit gehört, so müssen die kommentierenden Anmerkungen auch Informationen bringen, die dem Leser in Lateinamerika oder Japan wichtig, im deutschen Sprachraum aber überflüssig sein könnten. Freilich kann das für die akademisch-wissenschaftlichen Bücher Bonhoeffers, nämlich Band 1 und 2, nur in eingeschränktem Maße praktiziert werden, da sonst die Zahl der Anmerkungen ins Unabsehbare ansteigen würde. Im übrigen aber sollen die Bände dem interessierten Gemeindeglied wie dem Studenten, dem Teilnehmer an der Erwachsenenbildung wie dem Widerstandsforscher, dem Sucher nach religiöser Wahrheit wie dem geschichtlich Interessierten möglichst zugänglich sein und Bonhoeffer endlich auch verstärkten Eingang in die reguläre Theologenausbildung verschaffen. 
All denen, die durch ihre Mitarbeit den Start der „Dietrich Bonhoeffer Werke“ ermöglicht haben, sagen die Herausgeber ihren herzlichen Dank. Das kann an diesem Ort leider nicht namentlich geschehen. Die Liste der zu Nennenden würde zu lang. Wir wollen allerdings nicht unerwähnt lassen, daß ohne eine großzügige Stiftung von Herrn Heinrich Ludewig das Erscheinen der beiden ersten Bände zu diesem Zeitpunkt nicht möglich gewesen wäre und daß ohne erhebliche Druckkostenzuschüsse der Evangelischen Kirche in Berlin-Brandenburg und der Evangelischen Kirche der Union die Bände wesentlich teurer angeboten werden müßten. Der Chr. Kaiser Verlag setzt seine Tradition, Dietrich Bonhoeffers Werke herauszubringen, mit dem Wagnis dieser neuen Werkausgabe fort.

Dem Urteil des Lesers über das Werk, die Theologie und die Bedeutung Dietrich Bonhoeffers vorzugreifen, kann nicht die Aufgabe der Herausgeber sein. Jedoch wird mancher fragen, warum dieser Kreis, eine etwa zehn Jahre lange mühevolle Arbeit auf sich genommen hat, um dieses Werk neu herauszugeben. Der anfangs genannte Hinweis auf Bonhoeffers Aktualität für die Weltchristenheit deutet schon in die Richtung einer Antwort. Dies wird vielleicht dem nicht genügen, der fragt, welche theologische Zukunftsbedeutung die Bearbeiter selbst dem Werk Bonhoeffers beimessen. Da in einem Herausgeberkreis auch bei grundsätzlicher Übereinstimmung verschiedene Sichtweisen zusammenkommen, will ich für mich persönlich Auskunft geben.

Die Rezeption der Theologie Bonhoeffers scheint mir trotz vieler zum Teil hochqualifizierter Untersuchungen erst in den Anfängen zu stehen. Einer der Hauptgründe dafür liegt in dem fragmentarischen Charakter des Nachlasses dieses Mannes, der die letzten zwölf Jahre seines Lebens durch Kirchenkampf, Opposition und konspirativen Widerstand gegen den Nationalsozialismus in Atem gehalten wurde und schon als neununddreißig)ähriger sein Leben verlor. Die „Ethik“, die sein Lebenswerk werden sollte, liegt nur in Fragmenten vor. Die „Briefe und Aufzeichnungen aus der Haft", in denen sich die Umrisse 
eines überraschend neuartigen Entwurfes der Theologie abzeichneten, waren erste Versuche der Selbstverständigung, vor allem im brieflichen Gespräch mit dem Freunde Eberhard Bethge. Sie waren keineswegs in dieser Fơrm für die Veröffentlichung bestimmt. So muß der Interpret selbständig weiterdenken und Verbindungen herstellen, die der Autor, hätte er selbst nach 1945 publizieren können, vermutlich aufgezeigt hätte. Dieses Weiterdenken ist überaus reizvoll, aber auch schwer, soll es in verantwortlicher Weise geschehen. Man muß das ganze Werk Bonhoeffers dabei vor Augen haben, insbesondere die Dynamik seines theologischen Lebensweges.

Karl Barth, dieser große Calvinist, zu dem Dietrich Bonhoeffer als bewußter Lutheraner - bei allen Differenzen im einzelnen - unbeirrt stand, als Barth im Kirchenkampf isoliert wurde, bezeichnet in der Kirchlichen Dogmatik IV/2 1955 Bonhoeffers Buch „Nachfolge“ als „mit Abstand das Beste, was dazu geschrieben ist", und erklärt, daß er wohl versucht sein könnte, große Abschnitte dieses Buches „einfach als großes Zitat einzurücken, weil ich wirklich nicht der Meinung bin, etwas Besseres dazu sagen zu können, als da gesagt ist". Bonhoeffer selbst betrachtete 1944 dieses Buch als nur eine Station auf seinem theologischen Weg. Neue theologische Erkenntnisse kamen hinzu und drängten ihn zu einem umfassenderen Neuentwurf der Theologie, in den die Gedanken der "Nachfolge“ aufgehoben wurden.

In welchen Hinsichten wir inhaltlich Wichtiges, Unentbehrliches von Bonhoeffers Theologie zu erwarten haben, macht man sich am besten durch einen Vergleich klar. Ich wähle dazu die vier Systematischen Theologen, welche in dem von Carl Heinz Ratschow ab 1981 herausgegebenen „Handbuch Systematischer Theologie" zu den Klassikern unseres Jahrhunderts erhoben worden sind: Karl Barth, Paul Tillich, Werner Elert und Paul Althaus. Ihnen allen war vergönnt, in einer reichlich bemessenen Lebenszeit große Darstellungen ihrer Theologie vorzulegen. Elert und Althaus gelten bis heute als die maßgebenden Repräsentanten der Theologie des deutschen Luthertums im 20. Jahrhundert. Aber für das christlich-jüdische Gespräch, das nach dem Holocaust zu einer Notwendigkeit für uns Christen geworden ist, lassen sie uns ganz im Stich. Das 
Thema ,Judenfrage', zu dem sie sich freilich in den Jahren um 1933 ausgiebig und verhängnisvoll geäußert haben, kommt nach 1945 bei ihnen nicht mehr vor. Bonhoeffer hingegen hat nicht nur im April 1933 seine berühmte Stellungnahme „Die Kirche vor der Judenfrage" verfaßt, sondern konfrontierte in Lehrveranstaltungen, vor allem im Predigerseminar Finkenwalde, seine Kandidaten und Hörer beständig mit den biblischtheologischen Grundfragen dieses Problems. Das Thema war für ihn theologisch dringlich, und zugleich hat er sich, auf die Dauer unter großer eigener Gefahr, unablässig um praktische Hilfe für Juden ohne Rücksicht auf ihre religiöse Zugehörigkeit bemüht. Er las das Alte Testament ganz anders als Elert und Althaus. Seine theologischen Überlegungen berühren sich stark mit Barths späterer Israeltheologie.

Die Haftaufzeichnungen zum Problem der Mündigkeit der Welt und einer kommenden Religionslosigkeit gerieten in den sechziger Jahren in den Sog der Emanzipations- und Säkularisierungswelle. Einseitig naiv rezipiert, vermittelten sie ein verzerrtes Bild von dem Theologen Bonhoeffer. Dadurch wurde verdeckt, wie sehr eine tief durchdachte und zugleich lebensbezogene Christologie und Ekklesiologie das Zentrum von Bonhoeffers Werk ist. Was allerdings der Theologie Bonhoeffers ihre einzigartige Spannung gibt, das ist die Dichte, mit der er Wirklichkeitserfahrung und christologisch-ekklesiologische Erkenntnis zusammenbringt. Wenn er in den Briefen aus der Haft, sich selbstkritisch einbeziehend, der Bekennenden Kirche und Karl Barth den Vorwurf eines „Offenbarungspositivismus" machte, so war das nur die Kehrseite dieser Leidenschaft, Theologie nicht von der menschlichen Erfahrungswirklichkeit abzusondern, sie nicht in einen Ideenhimmel oder ein abstraktes Gedankensystem zu verwandeln, sondern sie vom Eindringen des Reiches Gottes in die Lebenszusammenhänge dieser Welt sprechen zu lassen. Für seine „Ethik“ schrieb er: „Die Wirklichkeit Gottes erschließt sich nicht anders als indem sie mich ganz in die Weltwirklichkeit hineinstellt, die Weltwirklichkeit aber finde ich immer schon getragen, angenommen, versöhnt in der Wirklichkeit Gottes vor. Das ist das Geheimnis der Offenbarung Gottes in dem Menschen Jesus Christus. Die christliche Ethik fragt nun nach dem Wirklichwerden dieser 
Gottes- und Weltwirklichkeit, die in Christus gegeben ist, in unserer Welt." Es ist hier nicht darzustellen, wie auch Barth in seiner späten Theologie weit über seine theologischen Schriften der dreißiger Jahre hinaus die Konfrontation von Offenbarungswirklichkeit und Weltwirklichkeit - zum Beispiel in politicis, etwa in der Frage atomarer Bewaffnung - herbeigeführt hat. Angesichts der reifen Gestalt von Barths Theologie erledigt sich zwar Bonhoeffers früher Vorwurf des Offenbarungspositivismus. Dennoch bleiben charakteristische Unterschiede. Bei Bonhoeffer war diese grundsätzliche Ausrichtung, dieses leidenschaftliche Sich-Bewegen des Christusglaubens auf die Weltwirklichkeit hin, schon ganz früh wirksam. Aber in voller Klarheit wurde es erst in seinen letzten Lebensjahren in der bedrängten Situation des am Widerstand Beteiligten expliziert, so zum Beispiel unter den Stichworten der Diesseitigkeit des Glaubens und der weltlichen Interpretation der biblischen Botschaft.

Das Wirklichkeitsverständnis von Elert und Althaus war, wenn man von der Theologischen Ethik des Politischen redet, gegen große Errungenschaften der Neuzeit gerichtet. Verfassungsstaat, Menschenrechte, Demokratie, Emanzipation zur rechtlichen Gleichheit aller Bürger galten ihnen als ,westliche Verirrungen und wurden auch nach 1945 nur mühsam von ihnen rezipiert. Insofern repräsentieren sie in hohem Maße das deutsche Neuluthertum vor der Mitte des zwanzigsten Jahrhunderts. Bonhoeffer erkannte die Gefahr dieser anti-neuzeitlichen Bindungen neulutherischer Theologie und bemühte sich gerade als lutherischer Theologe - um den Durchbruch der theologischen Ethik zu einem Wirklichkeits- und Politikverständnis, welches das faktisch vollzogene Mündigwerden der Welt, also eben die Neuzeit, begriff und verarbeitete. Mündigkeit hieß für ihn, daß die Welt die Gesetze, nach denen sie sich bewegt und mit deren Hilfe sie das Leben verantwortlich zu gestalten hat, zu durchschauen lernt, daß sie also die Möglichkeit der Selbststeuerung anstelle des schicksalhaft Getriebenwerdens ins Auge faßt. Daß ein solcher Schritt tiefgreifende Folgen für das theologische - und das heißt Bibel und Tradition neu auslegende - Gottesverständnis hat, brachte Bonhoeffer sofort zur Sprache. Nunmehr muß von Gottes Vorsehung und 
Weltregiment anders geredet werden als unter vorneuzeitlichen Bedingungen. Bonhoeffers Sprechen von der gewollten Ohnmacht Gottes in der Welt, seine Aufforderung an uns Christen, in der Fülle der Diesseitigkeit zu leben und darin die Leiden Gottes an seiner Welt mitzuleiden, machen das deutlich. Er drang damit tiefer in die Probleme der Säkularisierung ein als Friedrich Gogarten, der zwei Jahre nach der Veröffentlichung dieser Gedanken Bonhoeffers mit dem Buch „Verhängnis und Hoffnung der Neuzeit - Die Säkularisierung als theologisches Problem" 1953 gar nicht in den Blick bekam, wie sehr das Gottesverständnis und die Christologie durch das Mündigwerden der Welt - in all seiner gefährlichen Ambivalenz - betroffen wird.

Die Leidenschaft, mit der Bonhoeffer davon zu sprechen suchte, wie Gottes Wirklichkeit in ihrer Offenbarung durch Christus in die Weltwirklichkeit eindringt, trieb ihn an, die Weltwirklichkeit möglichst adäquat zu erfassen und in ihr verantwortlich zu handeln. Ich kenne keinen am konspirativen Widerstand gegen das nationalsozialistische Gewaltregime Beteiligten, der so engagiert und betroffen, so präzise und so schonungslos - auch gegen sich selbst - die Gewissenskonflikte, die menschlichen und die ethischen Probleme des Standhaltens gegen Hitler durchreflektiert hat wie Dietrich Bonhoeffer. Die „Rechenschaft an der Wende zum Jahre 1943“ mit der Überschrift „Nach zehn Jahren“ läßt das jeden heutigen Leser erkennen, obwohl in diesem Text, der damaligen Umstände wegen, viele der Sachverhalte chiffriert sind. Das zugleich schmerzhafte - die Frage eigener Schuld aufwerfende - und befreiende Bedenken des eigenen verantwortlichen Handelns gewinnt in Bonhoeffers Theologie eine Bedeutung wie wohl bei kaum einem anderen europäischen Theologen. Hier zeigt sich eine überraschende Nähe zu einigen Tendenzen in den Befreiungstheologien und in der Minjung-Theologie Asiens, die eine dem Schicksal der Niedrigen im Volk nahe Theologie sein will. Bonhoeffer gingen im Gefängnis die Augen dafür auf, was es bedeutet, aus der Sicht, von unten' den Glauben zu denken und in ihm zu leben. Von daher wurde ihm klar, daß auch das Verhältnis von theologischen Gedanken und christlichem Tun künftig anders bestimmt werden muß. Seine Generation, 
meinte Bonhoeffer, habe "zu stark in Gedanken gelebt“, habe zu sehr durch Bedenken im voraus das eigene Tun absichern wollen. In einer künftigen Generation werde das Denken nicht mehr „der Luxus des Zuschauers" sein dürfen; vielmehr werde das Denken der Verantwortungsbereitschaft dienen müssen, die dem nötig werdenden Wagnis nicht ausweicht. Gerade das hielt er für biblisch zentral angesichts der Evangelienworte, die dazu mahnen, den Willen Gottes zu tun, um so innezuwerden, daß Leben in der Nachfolge Christi Leben aus Gott ist (Matthäus 7,21; Johannes 7,17). Gewiß ist die Situation, in der Bonhoeffer lebte und dachte, nicht in unsere Gegenwart übertragbar. Daß aber Theologie sich immer wieder auf ihren Ursprung nicht in Gedanken, sondern im Tun des Beters und des verantwortlich Handelnden besinnen und daraus Konsequenzen ziehen soll und darf, das hat Dietrich Bonhoeffer uns in ungewöhnlicher Luzidität vorgelebt und vorgedacht.

Es wäre auch noch davon zu reden, wie tiefgreifend Bonhoeffer die Lehre von der Kirche und ihre Gestalt in Bewegung bringt. Aber das führt über die hier nur möglichen kurzen Hinweise hinaus. Sie sollten zeigen, daß wir ein volleres Verständnis und ein tieferes Wirken von Bonhoeffers Theologie nicht hinter uns, sondern größtenteils noch vor uns haben. Darum scheint mir die Neuausgabe der Werke nötig.

\section{III}

Eine große Vielfalt erwartet den Leser in Bonhoeffers Werken. Bonhoeffer fesselt nicht nur in seinen theologisch-programmatischen Schriften, sondern auch in den anderen Genera seiner Mitteilungen und Äußerungen. Die Entwürfe für Ansprachen im Kindergottesdienst zum Beispiel, die den Ausbildungsjahren entstammen, überraschen durch die unverwüstliche Frische ihrer religiösen Gedanken. In den Briefen zeigt sich schon früh eine bemerkenswerte Fähigkeit, sich dem Partner mitzuteilen und ohne Angst rückhaltlose Anfragen an ihn wie an die eigene Person auszutauschen. Die Bereitschaft, für sich und den Adressaten die vollen Konsequenzen auf sich zu nehmen, kündigt sich an. So führen die Briefe wie auch die Tagebücher 
direkt in die Ereignisse und Stimmungen, die Gedanken und die Hoffnungen der Jahre zwischen 1918 und 1945 ein. Zahlreich sind die Schriften und Ausarbeitungen zu besonderen Anlässen, insbesondere in der ökumenischen Arbeit, dem Kirchenkampf, der Theologenausbildung und - hier freilich in verschlüsselter Form - zur Existenz im Widerstand. Sie bringen theologische Grundsatzüberlegungen in unmittelbaren Kontakt mit den beteiligten Personen und den aktuellen Entscheidungen.

Die Rede- und Argumentationsweise Bonhoeffers ist deutlich der damaligen Zeit zuzuordnen. Manches klingt für den heutigen Leser zu autoritativ, zu apodiktisch. Aber auch junge Menschen empfinden, wenn sie sich mit Bonhoeffers Schriften befassen, daß sich in ihnen ein Mensch authentisch äußert. Bei Bonhoeffer behalten selbst Gelegenheitsschriften, die doch in der Regel schnell veralten, eine erstaunliche Brisanz. Das gilt für die Stellungnahme vom April 1933 zur „Judenfrage“, von der Klaus Scholder geschrieben hat: „Hier wurde mit unvergleichlicher Präzision die theologische Problematik staatlichen Handelns auf einen Begriff gebracht, der auch unter den veränderten Bedingungen der Gegenwart noch immer Gültigkeit besitzt. “ Es gilt ebensosehr für den im August 1934 vorgetragenen Aufruf zu einem ökumenischen Friedenskonzil der Kirche Jesu Christi, der im Sommer 1985 von Carl Friedrich von Weizsäcker erneuert worden ist.

Die geistlichen Texte, besonders Predigten und Meditationen, verweisen am dringlichsten auf das Zentrum eines Lebens in der Gemeinschaft mit Jesus Christus. Von ihnen geht Forderung und Trost, Wegweisung und Ermutigung aus und etwas, was man vielleicht mit dem Wort Erleuchtung charakterisieren darf. Hier wird die Sprache, je mehr Bonhoeffer sich von seiner akademischen Jugendzeit entfernt, immer freier von theologischen und kirchlichen Sprachkonventionen, entwickelt in sich selbst aber eine hohe Disziplin. So zupackend sie wirkt, so sicher weiß sie falsche Töne zu vermeiden und den nachdenklichen Leser zu gewinnen.

Die Neuausgabe ergreift die Chance, durch übersichtliche Zusammenstellung zeitlich zusammengehöriger Texte den Leser vor die „Polyphonie“ der Lebensäußerungen Bonhoeffers zu stellen. Dieses Wort benutzte Bonhoeffer gern in den Brie- 
fen aus der Haft. Er sah sich durch den Glauben ermächtigt, „mehrdimensional-polyphon“ zu denken und zu leben.

Heidelberg, im Advent 1985

Heinz Eduard Tödt für den Herausgeberkreis 
